Alina Concepción Alvarez*, Ivette Camayd Viera, Lisy Vento Buigues, Yanet Fernández Martínez, Eraida Hernández, Lilia Caridad Marín Padrón, Jacqueline Pérez Rodríguez and Giselle Gámez Torres

\title{
Reference values of plasma homocysteine in Cuban children and adults
}

https://doi.org/10.1515/labmed-2019-0195

Received November 19, 2019; accepted May 27, 2020;

published online August 7, 2020

\section{Abstract}

Objectives: Homocysteine (Hcy) is a nonessential amino acid, produced by the demethylation of methionine. High Hcy levels, or hyperhomocysteinemia, have been associated with genetic and multifactorial diseases. Hcy reference values may vary between different populations, as Hcy levels are affected by factors such as sex, age, diet, smoking, and coffee consumption. The estimation reference interval (RI) allows to establish the normal values of this marker in population. At present, these levels are unknown in Cuba. The aim of this work is to estimate the Hcy reference intervals in Cuban children and adults.

Methods: Total Hcy concentration was quantified by high performance liquid chromatography (HPLC) in plasma. Hcy levels were evaluated in samples from 507 healthy individuals (260 children, 247 adults).

Results: RIs were estimated by nonparametric methods. We found significant differences between both age groups, but we did not find significant differences between sexes, within these groups. The established ranges were 2.5614.55 $\mu \mathrm{M}$ and 3.63-17.19 $\mu \mathrm{M}$ for children and adults, respectively. Also, we observed a weak association between Hcy levels and age in both sex groups.

\footnotetext{
*Corresponding author: Alina Concepción Alvarez, Laboratory of Biochemistry Genetic, Center National of Medical Genetics, No.3102,146 Street, Cubanacan, Playa, 11600, Havana, Cuba, E-mail: alinaconcepcion@infomed.sld.cu Ivette Camayd Viera, Lilia Caridad Marín Padrón, Jacqueline Pérez Rodríguez and Giselle Gámez Torres: National Center of Medical Genetics, Havana, Cuba

Lisy Vento Buigues: Medical Surgical Research Center, Havana, Cuba Yanet Fernández Martínez: Pediatric Hospital “Juan Manuel Marquez", Havana, Cuba

Eraida Hernández: Carlos J Finlay’s Hospital, Havana, Cuba
}

Conclusions: This is the first study that assesses Hcy reference values in Cuban population. Our results will allow the introduction of Hcy as a biochemical marker in laboratory testing.

Keywords: Cuban individuals; nonparametric method; plasma; reference intervals; total homocysteine.

\section{Introduction}

Homocysteine (Hcy) is a sulfhydryl-containing amino acid produced by the demethylation of the methionine (Met). The Hcy metabolism involves remethylation or transsulfuration pathways and is a key element of the methylation cycle [1].

Deficiencies of the enzymes and cofactors acting in these metabolic pathways, are causes of genetic diseases such as classical homocystinuria, N(5,10)-methylenetetrahydrofolate reductase deficiency, homocistinuria combined with megaloblastic anemia and methylmalonic aciduria combined with homocystinuria [2]. These disorders are characterized by increased Hcy levels in plasma; therefore, the quantification of Hcy is included in the diagnosis guidelines [3]. Also, it has been associated with several multifactorial diseases [4-8].

In laboratory testing, the reference interval (RI) of a given analyte is a range drawn from a distribution of values. The data is generated by the quantification of the analyte in biological samples of healthy subjects. RIs are usually defined as the central $95 \%$ of a reference population. RIs vary depending on the biological sample, specific factors affecting the population under analysis and the laboratory analytical methods [9].

The reference values provide physicians with an interval to compare test results for a particular person. There are guidelines that describe how to establish RI such as the International Federation of Clinical Chemistry (IFCC) and the Clinical Laboratory Standards Institute (CLSI). The most used guidelines are the C28-A3 and EP28-A3c [10, 11]. The estimation of RI is performed when a new measurement procedure or technology change is introduced in the laboratory. 
There are several published reference ranges of total plasma Hcy [12]; however, Hcy reference values have not been assessed in the Cuban population. This situation limits the introduction of Hcy in the diagnosis of genetic diseases, as well as its use in research related to multifactorial diseases. Our laboratory recently introduced an Hcy quantitative method for the diagnosis of genetic diseases [13]. The aim of this work is to estimate the Hcy RIs in Cuban population.

\section{Materials and methods}

\section{Subjects}

All procedures performed during this research were in accordance with the Ethical Committee of our institution and The Code of Ethics of the World Medical Association (Declaration of Helsinki). A total of 507 apparently healthy individuals (260 children, 247 adults) from Havana were selected. All adult participants and the children legal tutors offered their informed consent.

Exclusion criteria in both age group (children and adult) were: (1) conditions such as renal failure, severe psoriasis, cancer, acute lymphoblastic leukemia, lupus erythematosus, diabetes mellitus, organ transplantation, obesity, acute inflammatory response, anemia, vitamin B12 deficiency or symptoms of malnutrition; (2) individuals treated with a prescribed medication that could interfere with Hcy metabolism (methotrexate, phenytoin, carbamazepine, colestipol, oral contraceptives with estrogen, cholestyramine, nicotinic acid, cyclosporine, hormone replacement therapy); (3) persons who referred smoking habit and (4) those who declared addiction to the consumption of alcoholic drinks.

\section{Samples}

Blood samples were collected after a fasting period of more than $6 \mathrm{~h}$. Venipuncture was performed using vacuum tubes with ethylenediaminetetraacetic acid (EDTA) as anticoagulant (Vacutainer, Portugal) and samples were refrigerated for no more than $8 \mathrm{~h}$ until processing. Plasma was separated by centrifugation at 35,000 g (Kukusan H-103N) for 10 min and stored at $-20^{\circ} \mathrm{C}$ until analysis.

\section{Method}

The quantification of total Hcy in plasma was performed by HPLC with fluorescence detection (Shimadzu, Japan). The method described by Concepción et al. [13] was used for sample treatment and chromatographic conditions.

\section{Statistical analysis}

All statistical analysis was performed using the R programming language (version 3.4.1, 2017). The data distribution was assessed using the Shapiro-Wilk test. Outliers were detected by the Van der Loo method, as it provides very robust results once the right distribution is found, it can get more than one outlier and identifies the outlier values by itself; that is, and the researcher does not have to fix the total of outliers [14]. Kruskal-Wallis test was used to analyze differences between groups. Spearman coefficient was determined between the variables age and Hcy concentration. According to the recommendations of the IFCC and CLSI [10, 11], we estimated the RIs by a nonparametric method using percentiles (2.5 and 97.5th) for each group. The $90 \%$ confidence intervals (CI) for percentiles were calculated using nonparametric bootstrapping with 5000 replications.

\section{Results}

More than 120 individuals were studied from both age groups (Table 1). Shapiro-Wilk test showed that Hcy concentrations did not follow a normal distribution ( $p<2.2 \mathrm{e}-6)$. A total of 15 outliers were identified and excluded from statistical analysis.

Plasmatic Hcy values showed higher levels in adults than in children (Figure 1) and significant differences between both age groups were demonstrated (Kruskal-Wallis test, $\mathrm{p}=4.86 \mathrm{e}-07)$. However, no significant differences were observed between the means of each sex group ( 0.52 for children and 1.06 for adult, with confidence intervals lower and upper of -1.30 and 0.26 for children, and -0.08 and 2.20 for adult).

The RIs were estimated by a nonparametric method. The 2.5 and 97.5th percentile values of total plasma Hcy level were 2.56 and $14.55 \mu \mathrm{mol} / \mathrm{L}$ in children, while in adults were 3.63 and $17.19 \mu \mathrm{mol} / \mathrm{L}$ (Table 2).

The analysis of correlation between Hcy levels and age showed a weak association between both variables for both sex groups, slightly higher in the female than in the male

Table 1: Number of individuals studied according to age and sex.

\begin{tabular}{lrr}
\hline Age, years & & Sex \\
\cline { 2 - 3 } & Female & Male \\
\hline $0-17$ & 127 & 133 \\
$\geq 18$ & 120 & 127 \\
Total & 247 & 260 \\
\hline
\end{tabular}




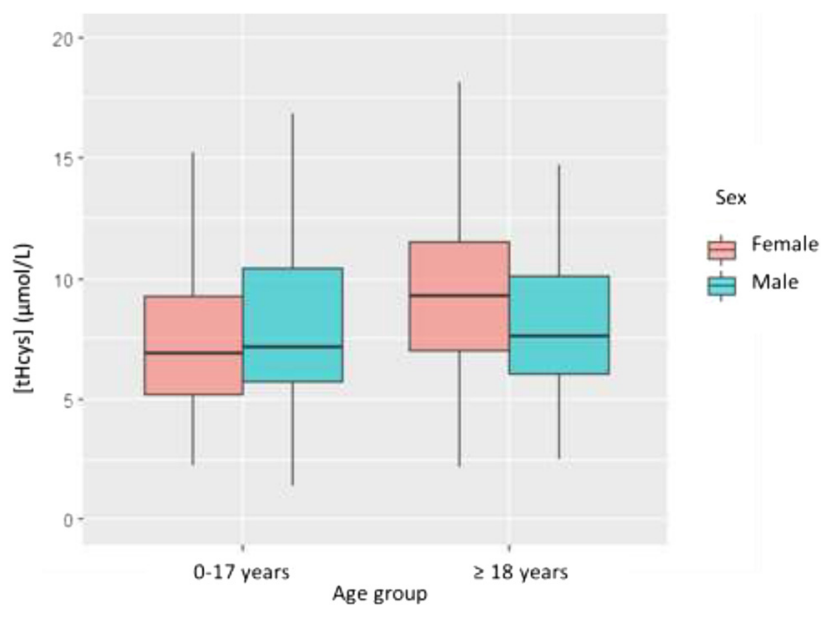

Figure 1: Homocysteine (Hcy) levels in plasma distributed by sex in children and adults.

thcy, total homocysteine concentration.

Table 2: Total homocysteine levels in plasma for age group.

\begin{tabular}{lrrrrr}
\hline Age, years & n & Mean & RI, $\boldsymbol{\mu m o l} / \mathbf{L}$ & Cl lower & Cl upper \\
\hline $0-17$ & 254 & 7.62 & $2.56-14.55$ & $1.99-2.98$ & $13.49-14.94$ \\
$\geq 18$ & 236 & 8.94 & $3.63-17.19$ & $3.02-3.84$ & $16.73-18.45$
\end{tabular}

n, number of individuals; SD, standard deviation; RI, reference interval 95\%; $\mathrm{Cl}$, confidence intervals $90 \%$.

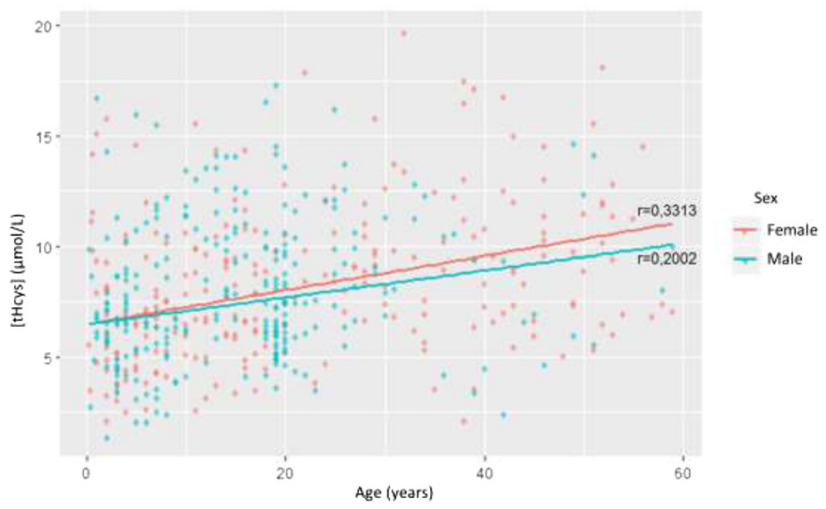

Figure 2: Total plasma Hcy levels in Cuban individuals as increase of age.

tHcy, total homocysteine concentration; r, Spearman coefficient.

group (Spearman coefficients of 0.2002 and 0.3313 for male and female, respectively; Figure 2).

\section{Discussion}

The participation of at least 120 individuals per age group allowed the estimation of the total Hcy RI in plasma, according to the international guidelines [10, 11]. Total plasma Hcy is considered a biochemical marker of genetic and multifactorial diseases [3]. Some authors remark the fact that the use of anticoagulant containing tubes to handle blood samples allows immediate quantification of plasma Hcy. Also, stability studies have suggested that even when serum is obtained under optimal conditions, Hcy levels are higher in this fluid than in plasma [15]. Besides, other laboratories have established RIs for serum Hcy. Comparison studies to assess the differences between the Hcy concentrations in plasma and serum, as well as the establishment of RIs of this analyte in serum, would be undoubtedly useful and could be carried out in the future.

The Shapiro-Wilk test and the quantile graphic representation of the data confirmed the absence of a normal distribution and suggested the presence of an exponential distribution. Other biological analytes have shown similar distributions. Furthermore, several analyses carried out to assess the RIs of Hcy in different populations have described non-normal distributions of their data [16, 17]. Therefore, nonparametric methods are more suited for RI estimation.

Our results show statistically significant differences between both age groups; although significant differences between both sexes within each age group could not be established. Most authors have reported differences between children and adult intervals, similar to our results. Also, published RIs for pediatric ages usually set a common range for male and female groups [18].

In Cuba, pediatric hospitals attend to children and adolescents (up to 18 years old). The detection of significant differences between pediatric age subgroups would require another study design, with a larger sample (again, 120 individuals per subgroup). Some authors describe RIs in children subgroups [20-23]; however, the correlation between total Hcy levels in plasma and age is weak, suggesting that the stratification of this group is unnecessary (Figure 2). Nonetheless, if the analysis of some specific subgroup is required for some specific reason, this research could be developed in the future.

When Hcy reference ranges are assessed in adults, is very common to find specific RIs for males and females. Conversely, some authors have reported differences between male and female groups [21, 22] while others could not corroborate such differences $[7,16]$. These variations have been related to the concentrations of estradiol, major female sex hormone, which has an inversely proportional relationship to the concentration of Hcy [18]. However, the role of this hormone in the metabolism of Hcy is poorly understood [19].

Our analysis showed a weak association between Hcy levels and age in both sex groups, slightly higher in the 
female group (Spearman coefficients of 0.2002 and 0.3313 for male and female, respectively; Figure 2). It has been widely accepted that Hcy levels increase with age, and hyperhomocysteinemia is more frequent in the elderly than in other age groups [15].

This research did not assess RIs of Hcy for specific subgroups within the adult population, such as postmenopausal women [24] or individuals older than 65 years [25]. According to the IFCC and CLSI guidelines, the evaluation of Hcy levels for certain conditions would require the stratification of the population into specific subgroups, and therefore the inclusion of at least 120 subjects per each class, as well as the design of appropriate questionnaires to collect more accurate information among participants. A more comprehensive analysis of Hcy levels in the Cuban population could be carried out in the future, according to the particularities of this country (for example, an aging population) and specific needs of the Cuban health system.

The mean values of Hcy levels were lower in children, with a difference of $1.32 \mu \mathrm{mol} / \mathrm{L}$ respect to the adults group (Table 2). In the ages of 0-17 years the obtained range was similar to those described by other authors who established intervals between 3 and $15 \mu \mathrm{mol} / \mathrm{L}$ in children and adolescents [12, 23, 26, 27]. For adults, published RIs showed a wider range (upto $17.19 \mu \mathrm{mol} / \mathrm{L}$ ). Our results are similar to the values described for Spanish [21] and Asian populations [22]. These similarities could be due to the Spanish genetic background present in our population, as a result of the process of admixture that occurred during the past centuries in our country.

Hyperhomocysteinemia is common to several monogenic and multifactorial diseases, as well as other physiological conditions. According to plasma levels, Hyperhomocysteinemia is classified in three types: mild, moderate, and severe. Mild and moderate types are mainly related to multifactorial diseases, while the severe type is generally associated to genetic defects such as cystathionine $\beta$-synthase deficiency $[3,28]$ or disorders of intracellular cobalamin metabolism [29]. Therefore, the estimation of RIs for plasma Hcy is crucial prior to the introduction of this marker in laboratory testing. The diagnosis and biochemical follow-up of disorders and conditions that occur with hyperhomocysteinemia requires a complete picture including clinical features and other biochemical and molecular tests.

\section{Conclusions}

This research allowed to establish the RIs of plasma Hcy for the Cuban population. Consequently, Hcy was included as a biochemical marker in the diagnosis and research of genetic diseases and other conditions that occur with hyperhomocysteinemia.

Research funding: This study was supported by the National Center of Medical Genetics, Havana, Cuba.

Author contributions: All authors have accepted responsibility for the entire content of this manuscript and approved its submission.

Competing interests: Authors state no conflict of interest. Informed consent: Informed consent was obtained from all individuals included in this study.

Ethical approval: All procedures performed during this research were in accordance with the Ethical Committee of our institution and The Code of Ethics of the World Medical Association (Declaration of Helsinki).

\section{References}

1. Lehninger L, Nelson D, Cox M. Lehninger principles of biochemistry, 5th ed. New York: W. H. Freeman; 2008.

2. University JH. Online mendelian inheritance in man; 2019. Available from: https://www.omim.org/ [Accessed: 4 Mar].

3. Morris A, Kožich V, Santra S, Andria G, Ben-Omran T, Chakrapani $A$, et al. Guidelines for the diagnosis and management of cystathionine beta-synthase deficiency. J Inherit Metab Dis 2017; 40:49-74.

4. Blom H. Overview of homocysteine and folate metabolism. With special references to cardiovascular disease and neural tube defects. J Inherit Metab Dis 2011;34:81.

5. Eloualid A, Abidi O, Charif M, El houate B, Benrahma $\mathrm{H}$, et al. Association of the MTHFR A1298C variant with unexplained severe male infertility. PLoS ONE 2012;7:e34111.

6. Schalinske K, Smazal A. Homocysteine imbalance: a pathological metabolic marker. Adv Nutr 2012;3:755-62.

7. Vučković B, Čabarkapa V, Ilić T, Salatić I, Crvenković Z, Mitić G. Clinical significance of determining plasma homocysteine: casecontrol study on arterial and venous thrombotic patients. Croat Med J 2013;54:480-8.

8. Ansari R, Mahta A, Mallack E, Luoa J. Hyperhomocysteinemia and neurologic disorders: a review. J Clin Neurol 2014;10:281-8.

9. Henny J, Vassault A, Boursier G, Vukasovic I, Brguljan P, Lohmander $M$, et al. Recommendation for the review of biological reference intervals in medical laboratories. Clin Chem Lab Med 2016;54:1893-900.

10. Clinical and Laboratory Standards Institute. Defining, establishing and verifying reference intervals in the clinical laboratory: approved guideline.CLSI document C28-A3, 28. 3rd ed PA: Clinical and Laboratory Standards Institute.: Wayne; 2008;61.

11. Horowitz G, Sousan A, Boyd J, Ceriotti F, Garg U, Horn P, et al. Defining, establishing, and verifying reference intervals in the clinical laboratory; approved guideline-Third edition, USA: CLSI document EP28-A3c; 2010. 
12. Wishart D, Tzur D, Knox C. Human metabolome database; 2019. Available from: https://www.hmdb.ca [Accessed 4 Feb].

13. Concepción A, Camayd I, Nuevas L. Validation of an HPLC method for total homocysteine quantification in plasma. Revista del Laboratorio Clínico 2016;9:40-7.

14. Van der Loo M. Distribution based outlier detection in univariate data. The Hague/Heerlen: Statistics Netherlands; 2010.

15. Refsum H, Smith A, Ueland P, Nexo E, Clarke R, McPartlin J, et al. Facts and recommendations about total homocysteine determinations: an expert opinion. Clin Chem 2004;50:3-32.

16. Brandão M, Pimentel F, Cardoso M. Serum homocysteine concentrations in Portuguese young adults. Acta Med Port 2011; 24:271-8.

17. Moon H, Whang D, Ko Y, Joo S, Yun Y, Hur M, et al. Reference interval and determinants of the serum homocysteine level in a Korean population. J Clin Lab Anal 2011;25:317-23.

18. Morris M, Jacques P, Selhub J, Rosenberg I. Homocysteinuria: an estrogen status indicators in the third National health and nutritional examination survey. Am J Epidemiol 2000;152:140-8.

19. Dierkes J, Jeckel A, Ambrosch A, Westphal S, Luley C, Boeing H. Factors explaining the difference of total homocysteine between men and women in the European investigation into cancer and nutrition potsdam study. Metabolism 2001;50:640-5.

20. Papandreou D, Mavromichalis I, Makedou A, Rousso I, Arvanitidou M. Reference range of total serum homocysteine level and dietary indexes in healthy Greek schoolchildren aged 6-15 years. Br J Nutr 2006;96:719-24.

21. Pijoán J, Garbizub I, Aguirre C. Intervalos de referencia poblacional y determinantes de la homocisteína plasmática. Med Clin (Barc) 2001;117:487-91.
22. Lahiri K, Datta H, Das H. Reference interval determination of total plasma homocysteine in an Indian population. Ind J Clin Biochem 2014;29:74-8.

23. Adeli K, Higgins V, Nieuwesteeg M, Raizman J, Chen Y, Wong S, et al. Complex reference values for endocrine and special chemistry biomarkers across pediatric, adult, and geriatric ages: establishment of robust pediatric and adult reference intervals on the basis of the Canadian health measures survey. Clin Chem 2015;61:1063-74.

24. Chillemi R, Simpore J, Persichilli S, Minucci A, D’Agata A, Musumeci S. Elevated levels of plasma homocysteine in postmenopausal women in Burkina Faso. Clin Chem Lab Med 2005;43:765-71.

25. Janson J, Galarza C, Muru A, Quintana I, Przygoda P, Waisman G, et al. Prevalence of hyperhomocysteinemia in an elderly population. Am J Hypert 2002;15:394-7.

26. Němec V, Bočkayová E, Kutílek S. Serum homocysteine levels in Czech children and adolescents. Acta Medica 2012;55: 87-90.

27. González G, Benser J, Breidenassel C, Albers U, Huybrechts I, Valtueña J, et al. Gender and age influence blood folate, vitamin B, vitamin B, and homocysteine levels in European adolescents: the Helena study. Nutr Res 2012;32: 817-26.

28. Sacharow S, Picker J, Levy H. Homocystinuria caused by cystathionine beta-synthase deficiency. Seattle (WA): University of Washington, Seattle; 1993-2020.

29. Sloan J, Carrillo C, Adams D, Venditti C. Disorders of intracellular cobalamin metabolism. In: GeneReviews [Internet]. Seattle (WA): University of Washington, Seattle; 1993-2020. 